\title{
POSTGRADO E INVESTIGACION EN LA ENSEÑANZA DE LA FISICA: EL MODELO DE LA UNIVERSIDAD FEDERAL DEL RIO GRANDE DEL SUR
}

MOREIRA, M.A.

Cornell University. Department of Education.

\section{SUMMARY}

In this paper the model of graduate program in physics education adopted at the Federal University of Rio Grande do Sul (UFRGS/Brasil) is described and some information about the Physics Education Group of the Institute of Physics of this University is given.

\section{RESUMEN HISTORICO}

El postgrado en enseñanza de la Fisica en el Instituto de Fisica de la UFRGS empezó en 1968 juntamente con la creación del curso de postgrado en Física de esa Universidad.

En esa época ya existía en el Instituto un grupo de profesores que tenía mayor interés en la enseñanza de la Fisica y en la investigación en esa área que en la investigación en Física propiamente dicha.

El postgrado en enseñanza de la Física fue entonces creado como opción que atendiese los intereses de ese grupo. La primera tesis de maestría en ese campo fue concluida en fines de 1971. A partir de ahí, hasta 1976, los otros miembros del grupo inicial, los cuales eran todos profesores de la UFRGS, fueron también concluyendo sus maestrias.

En 1977 fueron aceptados los primeros estudiantes de otras universidades, en particular a través del Programa Institucional de Capacitación Docente $y$, desde entonces, el postgrado en enseñanza de la Física pasó a atender principalmente candidatos de fuera de la UFRGS, inclusive del extranjero.

Al mismo tiempo se cuidó la continuidad, a nivel de doctorado en ensef̂anza de las ciencias, de la formación académica del grupo inicial, estableciéndose con ese objetivo un intercambio informal con la Universidad de Cornell, en los Estados Unidos, donde tres miembros de ese grupo hicieron el doctorado.

\section{EL MODELO ADOPTADO}

El modelo de postgrado en enseñanza de la Física de la UFRGS, esquematizado en la figura I presentada al final de ese trabajo, tiene las siguientes caracteristicas básicas:

1. La enseñanza es una de las áreas de concentración de la Maestría en Física de la UFRGS. Por tanto, el título obtenido es la Maestría en Física. (Las otras áreas son Física Experimental, Física Teórica, Astrofísica y Física Aplicada). Es decir, no se trata de una Maestría en Enseñanza de las Ciencias sino de una Maestria en Física con énfasis en la enseñanza de la Física.

2. El programa es desarrollado enteramente en el Instituto de Física, no habiendo, por tanto, convenio con la Facultad de Educación. Al comienzo, la parte de ensefianza del programa era dictada por especialistas de la Facultad de Educación, contratados por el Instituto, puesto que el postgrado en Física es anterior al postgrado en Educación en la UFRGS. Las primeras tesis de maestria en el área de enseñanza de la Física fueron dirigidas por físicos que tenían interès en esa área. Posteriormente, profesores del Instituto con doctorado en enseñanza de las ciencias tomaron a su cargo las asignaturas correspondientes a la parte educacional del programa asi como la dirección de las tesis.

3. Del conjunto de ocho asignaturas que totalizan los 32 créditos necesarios a la obtención del titulo, cuatro son obligatorias y comunes a todas las áreas de concentración: 
- Mecánica clásica

- Mecánica Estadística

- Mecánica Cuántica

- Teoría Electromagnética

Cada una de esas asignaturas tiene cuatro clases teóricas semanales y corresponde a cuatro créditos.

Las otras asignaturas para la área de enseñanza son las siguientes:

- Métodos de Instrucción y de Investigación en Enseñanza de la Física I y II.

- El laboratorio en la Enseñanza de la Física.

- Teorias del Aprendizaje Aplicadas a la Enseñanza

de la Fisica u otra asignatura eligida por el estudiante en Física o Edıcación.

En las asignaturas de «Métodos I y II» son abordadas, en particular, la metodología de la enseñanza superior y la metodología de la investigación en educación. La asignatura de «Laboratorio", como sugiere su propio nombre, enfoca la enseñanza de la Física Experimental, especialmente a nivel universitario básico. A su vez, la asignatura de "Teorias", enfoca las propuestas de B.F. Skinner, R. Gagné, J. Bruner, J. Piaget, D. Ausubel y $C$. Rogers como sistemas teóricos de referencia para la organización de la enseñanza de la Física y para la investigación en esa área. A veces, esa asignatura no es ofrecida en un determinado semestre o el estudiante opta por cursar otra asignatura en Física o en Educación (ent el curso de postgrado en Educación con el acuerdo de la Coordinación de ese Curso).

4. Además de las asignaturas mencionadas anteriormente, la maestría en enseñanza tiene aún como requisito básico el desarrollo de un proyecto de investigación en enseñanza de la Física, preferentemente a nivel universitario introductorio, que conduce a una tesis de maestría. Los requisitos adicionales son un examen sobre lectura e interpretación de textos científicos en inglés y la asignatura Estudio de Problemas brasileños.

5. Se trata de un programa que desde el comienzo ha sido mantenido en pequeña escala y que seguirá con esa característica en el futuro, o sea, no hay planes de implantación de un doctorado en enseñanza de la Física en este Instituto. Esa caracteristica del programa es coherente con el hecho de que la ensentanza es solamente una de las áreas de concentración de la Maestría en Física.

6. El programo está volcado principalmente hacia la enseñanza universitaria básica, pero no excluye otros niveles de actuación, y tiene por objetivo la formación del profesor y del investigador en enseñanza de la Física. Se aceptan graduados en Fisica así como profesionales de otras áreas que tengan los prerrequisitos pertinentes.

\section{ALGUNOS PRESUPUESTOS}

La filosofía del programa es que una sólida formación en Fisica aliada al conocimiento de técricas de enseñanza, de investigación en enseñanza y de teorias del aprendizaje y del desarrollo cognoscitivo son condiciones indispensables para la formación del investigador en enseñanza de la Fisica.

Tiene también en su base la premisa de que la maestría en enseñanza de la Física no debe ser una alterna. tiva para quien no obtuvo éxito en otras áreas de la maestria en Física. Los requisitos deben ser exactamente los mismos de las otras áreas, inclusive las asignaturas obligatorias.

En términos de enseñanza, pone énfasis en el aprendizaje de contenido, en la actividad experimental y en el uso de referenciales teóricos en la organización de la instrucción.

\section{TESIS YA CONCLUIDAS}

A continuación es presentada una lista completa de todas las tesis de maestría en enseñanza de la Fisica concluidas desde la implantación del programa en la UFRGS, totalizando 26 trabajos completados hasta marzo de 1986. Considerando que la primera tesis fue presentada en 1972 este total da un promedio de casi dos maestrias por año, to cual corrobora el carácter de pequeña escala que tiene el programa.

Esta lista de tesis da también una idea de la naturaleza de los trabajos desarrollados. Se puede observar claramente una énfasis inicial en aspectos metodológicos de la enseñanza que, progresivamente, fue substituida por un enfoque cognoscitivo. Paralelamente fueron también realizados unos cuantos estudios en el campo de la enseñanza de laboratorio.

Estas tesis están todas en portugués y de la gran mayoria hay solamente un ejemplar en la biblioteca del Instituto. Sin embargo, casi todos sus resumenes ya fueron publicados en Enseñanza de las Ciencias (1985, Vol. 3 (1), 63-66 y 1986, Vol. 4 (1), 80-81).

1. Moreria, Marco A. «La organización de la enseñanza de la Física en el ciclo básico de la universidad», 1972.

2. Schreiner, Wido H., «Instrucción programada en Física vía televisión», 1973.

3. Axt, Rolando, «El uso de un minicomputador como recurso de aprendizaje en experiencias simuladas", 1973.

4. Buchweitz, Bernardo, «Estudio sobre los Métodos Keller, Audiotutorial y Estudio Dirigido en Física», 1975.

5. Levandowski, Carlos E., «El sistema Audiotutorial en la enseñanza de Física General en la universidad", 1975.

6. Dionisio, Paulo H., «El método Keller y su aplicación en la ensetianza de Física General en la universidad», 1976. 
7. Silveria, Fernando L., "La influencia de la estructura cognoscitiva en el aprendizaje de Físicas, 1976.

8. Zawislak, Beatriz M.M., "La enseñanza individualizada en Fisica y sus efectos en el aprendizaje», 1976.

9. Salomão, M. Rejane, «Método laboratorial para el estudio de radiaciones electromagnéticas», 1977.

10. Santos, Carlos A., «Aplicación del escalamiento multidimensional y del análisis de agrupamientos jerárquicos en el mapeamiento cognoscitivo de conceplos físicos), 1978.

11. Gonçalves, Ennio S., «Laboratorio estructurado versus no estructurado: un estudio comparativo en un curso de Física General», 1979.

12. Costa, Regina C., «Estudio comparativo de la esiructura del contenido y la estructura cognoscitiva del profesor y del alumnos, 1980.

13. Sousa, Célia M.S.G., «Pseudo-organizadores previos como recursos facilitadores del aprendizaje en Fisicas, 1980.

14. Peduzzi, Luis O.Q., "Dos estudios sobre la solución de problemas de Fisica a nivel universitario básico: el efecto de una estrategia y la influencia de la estructura cognoscitivan, 1980 .

15. Lima, Augusto O., «Un aborde ausubeliano para la organización de! contenido en Termodinámica y Teoría Cinéticá de los gases a nivel de Física Generalı, 1981 .

16. Souza, Helson F., «Mapeamiento del contenido y mapeamiento cognoscitivo: un estudio incluyendo dos abordajes en la organización del contenidon, 1981.

17. Peduzzi, Sonia S., «Un aborde ausubelino en la enseñanza de Electricidad y Magnetismo a nivel univer. sitario básico», 1981 .

18. Passos, Antonto M.F., "Un estudio sobre la ensenanza de laboratorio a nivel universitario básico", 1981.

19. Zamora C., Sergio P., «Influencia del conocimiento previo sobre el desempeño del alumno en un curso de Física General individualizadon, 1981.

20. Farias, Antonio J.O., «Mapeamiento cognoscitivo en un curso individualizado: un estudio sobre el efecto del aborde al contenidom, 1982.

21. Ahumada G., Waldo E., «Mapas conceptuales como instrumento para investigar la estructura cognoscitiva del alumno", 1983.

22. Porto, Antonio V., «Actividades experimentales para la enseñanza de Mecánica de Fluidos a nivel universitario básicon, 1983 .

23. Gobara, Shirley T., «Mapas conceptuales como instrumentos didácticos en la enseñanza de la Física», 1984.

24. Santos, Arion C.K., "Un estudio sobre la enseñanza

ENSEN̄ANZA DE LAS CIENCIAS, 1987, 5 (1) de laboratorio de Fisica en escuelas de $2^{\circ}$ grado de Porto Alegre», 1985.

25. Dominguez D., M. Eugenia, «Detección de algunos conceptos intuitivos en Electricidad a través de entrevistas clínicas», 1985.

26. Jamett C., Hernan D., «Laboratorio de Física: un análisis del curriculum y del aprendizaje», 1985.

27. Silva, Laércio E.F., «Concepciones espontáneas en Termodinámica: un estudio en un curso universitario, utilizando entrevistas clinicas, 1986.

\section{EL GRUPO DE ENSEÑANZA}

La historia del grupo de enseñanza de la Fisica del Instituto de Fisica de la UFRGS, en ciertos aspectos, se confunde con la historia del postgrado en ensenanza de la Fisica en ese Instituto descrita hasta este punto. Como ya hemos dicho el área de concentración en enseñanza, presente desde la implantación de la Maestria en Fisica en la UFRGS, fue creada, inicialmente, a fin de posibilitar la formación postgraduada a un grupo de profesores del Instituto cuyo mayor interés era la enseñanza de la Física. Las primeras maestrias en esa área fueron concluidas por esos profesores, muchos de los cuales optaron definitivamente por la enseñan$\mathrm{za}$, profundizando en el asunto; algunos, inclusive, hicieron el doctorado en el exterior y pasaron a construir el grupo conocido como Grupo de Enseñanza.

Este grupo está actualmente formado por siete profe. sores, tres con maestrias en enseñanza de la Física y doctorado en enseñanza de las ciencias, dos con maestría en enseñanza de la Física, uno con maestría en Educación y uno con especialización en enseñanza de la Fisica, seis de los cuales trabajan a tiempo completo en el Instituto. Son también miembros del grupo, en general, cinco o seis estudiantes de postgrado, brasileftos y de otros paises latinoamericanos.

Dos son las líneas básicas de trabajo del grupo, desarrolladas en estrecha vinculación con el programa de postgrado en ensentanza de la Física:

5.1. Enseñanza de laboratorio. Producción y prueba de nuevos experimentos, aparatos y manuales para la enseftanza de laboratorio a nivel universitario básico; producción y prueba de conjuntos de aparatos y manuales para maestros para la enseñanza de laboratorio de Fisica en la escuela secundaria; diferentes abordes a la enseñanza de laboratorio; análisis epistemológico de experimentos de laboratorio.

5.2. Aprendizaje de conceptos físicos. Mapeamiento cognoscitivo según diferentes técnicas; la teoría del aprendizaje de David Ausubel como sistema de referencia para la investigación y la organización de la enseñanza; mapas conceptuales como instrumentos didácticos, de evaluación y de análisis del curriculum; investigación de conceptos espontáneos a través de entrevistas clínicas y de tests elaborados con base en las 
entrevistas; búsqueda de estrategias que teniendo como punto de partida el conocimiento previo del alumno conduzcan al cambio conceptual.

Las fuentes de financiación de estas actividades de investigación en enseñanza son, en general, las mismas que apoyan la investigación en Física desarrollada en el Instituto, principalmente la FINEP (Financiadora de Estudios y Proyectos) una agencia del gobierno. Por cjemplo, cuando el Instituto envía a la FINEP su plan bi-anual de investigación, la investigación en enseñanza de la Física es incluida como uno de los subprojectos de ese plan. Por otro lado, becas de estudio para estudiantes de postgrado brasileños o becas de investigación para miembros del grupo son dadas por dos otras agencias del gobierno, el $\mathrm{CNPq}$ (Consejo Nacional de Desarrollo Científico y Tecnológico) y la CAPES (Coordinación de Perfeccionamiento de Personal de Nivel Superior). La UFRGS, naturalmente, provee sueldos, espacio físico y otras condiciones materiales.

\section{PERSPECTIVAS FUTURAS}

Por tratarse de un grupo de investigación en enseñanza dentro de una institución donde, primordialmente, se hace investigación en Física, hubo, y aún hay, la ne- cesidad de luchar por un espacio institucional, pero las perspectivas son que el grupo continúe desarrollando sus actividades normalmente en los próximos años.

Un fenómeno relativamente reciente, pero que probablemente representa una tendencia futura es el trabajo conjunto entre miembros del grupo de enseñanza e investigadores en Física. Por ejemplo, un proyecto en el área de enseñanza de la Física con microcomputadoras y otro en el campo de la enseñanza de laboratorio en la escuela primaria están siendo actualmente desarrollados con la cooperación de investigadores que hasta ahora hacian solamente investigación en Física.

Se pretende también mantener el vinculo informal existente con el programa de enseñanza de las ciencias de la Universidad de Cornell. De acuerdo con esa línea, desde septiembre de 1986 el autor de este trabajo se encuentra actualmente en Cornell como visitante, para trabajar con el grupo del Profesor Joseph Novak durante dos años.

Con relación al postgrado en ensenanza de la Física, se espera mantenerlo en pequeña escala y como área de concentración de la Maestría en Física, incluso en caso de que llegue a crearse en la UFRGS una Maestría en Ensertanza de las Ciencias pues, como fue dicho antes, el enfoque es diferente.

Una visión esqucmatizada del modelo de maestria en enseñanza de la Fisica adoptado actualmente en la UFRGS (\$986)

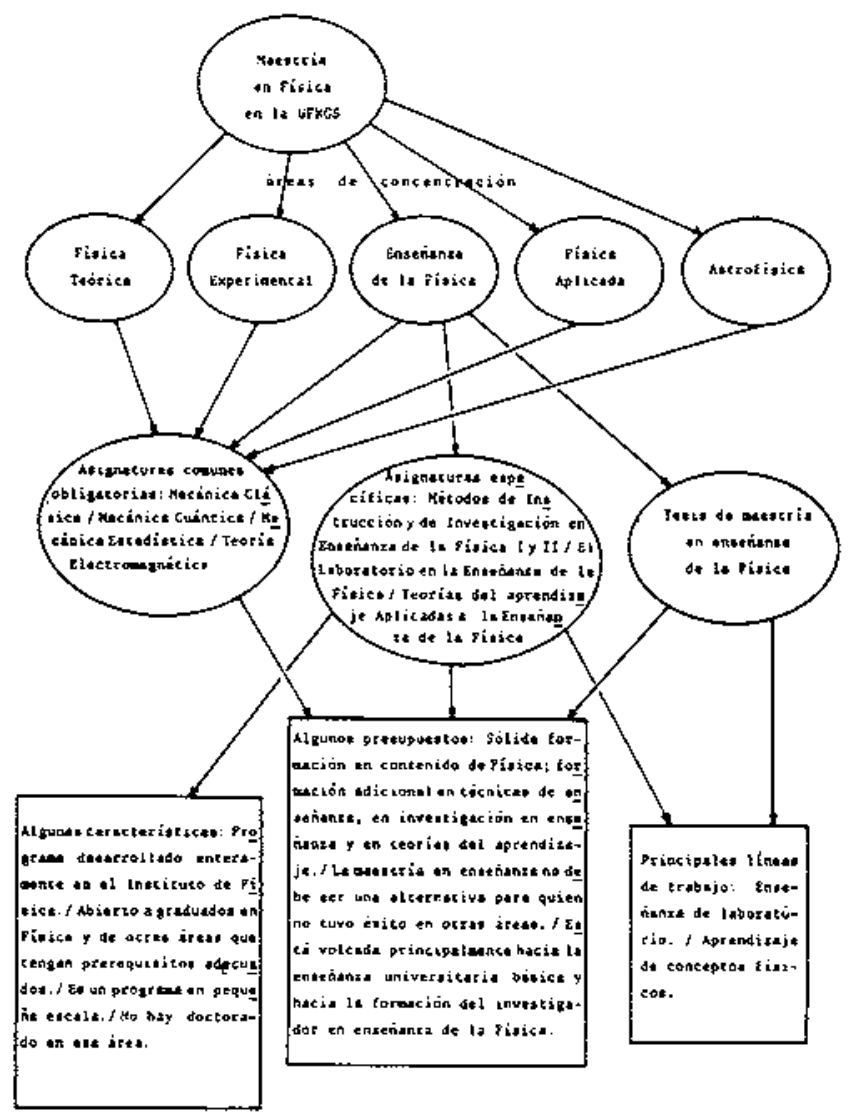

